\title{
COLLOQUES ET CONGRES
}

Le testicule humain. Son rôle dans la reproduction et la sexualité.

22-24 Avril 1998 - PISE (Italie)

Contact : $\quad$ Fabrizio MENCHINI-FABRIS

Centre d'Andrologie, Université de Pise

Via Roma 67, 56126 Pisa, Italie

Tél. 39.50.53.34.04; Fax 39.50.55.00.33

L'Andrologie dans les années 90, 4ème édition.

Symposium international sur l'infertilité masculine et l'Assistance médicale à la procréation.

$$
\text { 22-25 Avril } 1998 \text { - GAND (Belgique) }
$$

Contact : Dr. W.OMBELET

Genk Institute for Fertility Technology

Zol Campus St Jan. Schiepse Bos 6, B-3600 Genk, Belgique

Tél. 32.75.53.07.39 ; Fax 32.89.32.36.91

E-mail : gyneco.genk.gift@tornado.be

Vème Congrès de la Société marocaine d'andrologie et de sexologie.

30 Avril - 2 Mai FES (Maroc)

Contacts : 1) Dr Aziz SMIRES-BENNANI

44, rue Abou Ahdellah Nafii

20100 Maârif - Casablanca

Tél. 212.2.25.95.73 ; Fax 212.2.98.60.51

2) Dr. M.SCHOUMAN

164, Avenue Charles de Gaulle - 92200 Neuilly

Tél. 01.47.47.02.02 ; Fax 01.47.47.47.34 - Mél : Schouman@infonie.fr

Réunion annuelle de la Société pour l'étude de l'impuissance

30 Mai 1998 SAN DIEGO (USA)

Contact : $\quad$ SSMR, 111 North Plaza Drive, suite 550

Schaumburg, Illinois 60173, USA

Tél. 847.517.7225 
8ème Symposium international sur la spermiologie

11-22 Août 1998 MONTREAL (Canada)

Contact : $\quad$ 8th ISS Secretariat, 1224 Stanley Streat, Unité 211, Montréal, Québec, Canada, H3 B257

Tel. 514.878.2530 ; Fax 514.878.2532

8ème réunion mondiale sur la recherche sur l'impuissance et 11ème symposium sur la revascularisation du corps caverneux

$$
\text { 25-28 Août } 1998 \text { AMSTERDAM (Hollande) }
$$

Contact : Congress Secretariat, Ms Marianne Mulder

Département d'Urologie, Hôpital Universitaire de Nijmegen, P.O. Box 9101,65000HB Nijmegen, Hollande

Tél. 31.24.3613920 ; Fax 31.24.354.1031 - Mél : m.mulder@uro.azn.nl

\section{XVème CONGRES DE LA SOCIETE D'ANDROLOGIE DE LANGUE FRANCAISE}

\section{0-12 Décembre 1998, LYON}

Préprogramme :

- Génétique des infertilités

- Microdélétions sur le chromosome Y

- Cryptorchidies :

- Aspects clinicobiologiques

- Gènes impliqués

- Perturbateurs endocriniens

- Le plasma séminal

- Les gènes de la spermatogenèse

- Apports et limites de la transgenèse

- Stérilités et anomalies génétiques

- Clonage des gènes testiculaires humains : intérêt pour l'étude des stérilités

- Table ronde : Recherche et étude de la Stérilité

- Biopsie testiculaire

- Pharmaco-toxicologie des réactions sexuelles

Contact : Dr M.Benahmed U.INSERM 407

Faculté de Médecine Lyon-Sud BP 12

69921 OULLINS Cedex

Tél. 04.78.86.16.06 - Fax 04.78.86.59.22 